\title{
La institucionalidad y su incidencia en el fomento de los clústeres locales: Evidencia para Ecuador periodo 2007-2017
}

\section{Institutionality and its impact on the promotion of local clusters: Evidence for Ecuador period 2007-2017}

\author{
Daniel Disyorque Peñaherrera Patiño \\ https://orcid.org/0000-0001-9216-8561 \\ Universidad de Guayaquil, Ecuador
}

Autor por correspondencia: daniel.penaherrerapa@ug.edu.ec

Fecha de recepción: 26 de septiembre de 2019 - Fecha de aceptación: 27 de febrero de 2020

\begin{abstract}
Resumen
Este trabajo describe los principales cambios suscitados en Ecuador en el periodo 2007-2017, relacionados al fortalecimiento de los clústeres locales (CL) y las cadenas de valor locales (CV) analizando su incidencia en el nivel de competitividad del país. El objetivo, es relacionar las reformas institucionales con los indicadores económicos alcanzados en materia de competitividad, tomando como referencia la evolución en la posición competitiva alcanzada en el índice de competitividad global. Se ha utilizado una metodología de investigación descriptiva y aplicada con enfoque mixto, que parte desde la teoría para llegar a causalidades y conclusiones sobre el fenómeno de estudio. El trabajo introduce un modelo teórico denominado, esquema de política económica estructural, el cual se orienta al fomento del desarrollo productivo a través de las políticas económicas estructurales, como base (causa) que genera los incrementos del ingreso y del gasto (efectos) en la economía. Los resultados demuestran que los cambios institucionales incidieron en el mejoramiento competitivo de 31 puestos en la posición del Ecuador periodo 2013-2014 respecto al periodo 2007-2008. Desde el periodo 2015-2016 y hasta el periodo 2017-2018 se puede evidenciar un retroceso significativo de 26 posiciones, lo cual expone la vulnerabilidad y dependencia de la estructura productiva ecuatoriana.
\end{abstract}

Palabras claves: clústeres locales; cadenas de valor; crecimiento económico; desarrollo productivo; competitividad; institucionalidad

\begin{abstract}
This work describes the main changes in Ecuador in the 2007-2017 period, related to the strengthening of local clusters (CL) and local value chains (CV) analyzing their impact on the country's level of competitiveness. The objective is to relate the institutional reforms with the economic indicators achieved in terms of competitiveness, taking as reference the evolution in the competitive position reached in the global competitiveness index. A descriptive and applied research methodology with a mixed approach has been used, which starts from the theory to arrive at causalities and conclusions about the study phenomenon. The work introduces a theoretical model called, structural economic policy scheme, which is oriented to the promotion of productive development through structural economic policies, as the basis (cause) that generates increases in income and expenditure (effects) in the economy. The results show that the institutional changes affected positive the competitive improvement of 31 positions in the
\end{abstract}


ranking 2013-2014 with respect to the 2007-2008 period. From the 2015-2016 period to the 2017-2018 period, a significant setback of 26 positions can be evidenced, which exposes the vulnerability and dependence of the Ecuadorian productive structure.

Key words: local clusters; value chains; productive development; competitiveness; institutionality

\section{Introducción}

Para poder aumentar la competitividad de un país es importante poder contar con un entorno institucional adecuado, que condicione de manera positiva las relaciones entre los agentes económicos. El entorno institucional está determinado principalmente por el marco jurídico y administrativo en el que los individuos, las empresas y los gobiernos interactúan para generar ingresos y riqueza en la economía.

El presente trabajo busca incorporar al debate académico, la profundización en el estudio de los elementos institucionales que inciden favorablemente en la presencia y construcción de una política económica estructural orientada al cambio del modelo productivo ecuatoriano, que contribuya al desarrollo de los CL y las CV y su relación con la evolución de la competitividad de la economía ecuatoriana. El objetivo es relacionar las reformas institucionales y legales implementadas en Ecuador en el periodo de referencia, con la evolución de los resultados económicos obtenidos en materia de competitividad e institucionalidad, de tal manera de poder aumentar la comprensión sobre el rol de las instituciones para condicionar los resultados económicos esperados.

\section{Marco teórico}

Para poder realizar un análisis ordenado al respecto de los principales aspectos institucionales que contribuyen al desarrollo de una política económica estructural orientada al cambio del modelo productivo ecuatoriano, al fortalecimiento de los clústeres locales (CL) y las cadenas de valor locales (CV), es necesario estructurar un marco teórico básico, que permita orientar de forma ordenada, los principales conceptos que tienen una incidencia directa para el análisis del tema planteado. Es importante recalcar que la literatura científica al respecto de los aspectos institucionales, clústeres y cadenas de valor, han tenido una evolución importante en las últimas décadas y abarcan diversas perspectivas, las mismas que, son complementarias y tienen alcances importantes en la economía en su conjunto.

Para la revisión del marco teórico la estructura será dividida en sub-apartados de los conceptos claves, de tal manera, de poder darle un orden argumental a la revisión realizada.

Los subapartados son los siguientes:

i. Institucionalidad y nueva economía institucional (NEI).

ii. Política económica estructural (PEE).

iii. Competitividad y su relación con las políticas estructurales.

iv. Principales teorías económicas aplicadas al fomento productivo.

En la revisión teórica de los siguientes subapartados, se plantea definir los conceptos claves, la importancia y principales alcances de los mismos, de acuerdo con el planteamiento de la propuesta de investigación. 


\section{Institucionalidad y nueva economía institucional (NEI)}

El estudio y análisis de cómo las instituciones generan condiciones favorables y cómo interactúan con soluciones organizativas para el mejoramiento de la eficiencia económica, es de vital importancia para el diagnóstico, diseño, implementación y evaluación de políticas públicas e institucionales.

El otorgamiento del premio nobel de Economía a cuatro destacados economistas como es el caso de Ronald Coase (1991), Douglas North (1993), Oliver Williamson (2009) y Elinor Ostrom (2009) ha permitido el reconocimiento y protagonismo del nuevo enfoque institucional enfatizándose el hecho de que los cambios institucionales, incluso son más importantes que los cambios tecnológicos como base para explicar el desarrollo económico y la competitividad de un país.

North (1990) definió a las instituciones como las reglas formales, normas informales y mecanismos de cumplimiento y explicó cómo esas reglas de juego determinan el volumen de los costes de transacción existentes, y por tanto el nivel de eficiencia alcanzable en la economía. El rol de las instituciones de acuerdo con este enfoque es que definen y limitan el conjunto de elecciones de los individuos, estableciendo las reglas según las que se desarrolla el juego. En este sentido, cada sociedad tendrá que construir unas reglas de juego las mismas que serán las que determinen el costo de realizar las transacciones y por otra parte facilitarán o dificultarán las transacciones económicas.

De acuerdo con Caballero \& Garza (2003) el nuevo institucionalismo permite un mejor conocimiento e interpretación del papel de las instituciones en una amplia variedad de fenómenos económicos, políticos y sociales. La NEI busca como finalidad, explicar la importancia de las instituciones en la vida social, tomando como base un lenguaje económico, pero al mismo tiempo, integrando conceptos de otras disciplinas como el derecho, la ciencia política, la sociología, la historia, o la antropología.

El enfoque institucional se sustenta en el hecho de que las instituciones condicionan los hechos económicos, en concordancia con este planteamiento, Acemoglu \& Robinson (2012) indican que el éxito económico de los países difiere debido a las diferencias entre sus instituciones, a las reglas que influyen en cómo funciona la economía y a los incentivos que motivan a las personas. Estos autores dividen a las instituciones económicas y políticas en inclusivas y extractivas, las primeras crean los incentivos y oportunidades para promover las actividades empresariales en la sociedad, reparten el poder ampliamente en la sociedad y lo limitan son pluralistas; y las instituciones extractivas no.

En líneas generales las instituciones trascienden los intereses individuales y su propósito es que todos los agentes, actores sociales y económicos, alcancen beneficios económicos y su funcionamiento está asociado a la elaboración de leyes, reglas o normas que buscan hacer eficiente la interacción económica.

\section{Política económica estructural (PEE)}

La política económica estructural (PEE) se puede definir como aquel conjunto de medidas, estrategias, directrices, lineamientos, planes e iniciativas enfocadas a la transformación productiva de una economía. 
Este tipo de políticas afectan directamente a la gobernanza y la sostenibilidad del desarrollo económico, puesto que permite la mejora de la competitividad de las empresas y los sectores donde estas operan, orientando su acción en el marco territorial en el que estos despliegan sus actividades económicas (Sánchez, Ochando y Torrejón, 2018). Cuando se habla de políticas económicas estructurales se vinculan otras concepciones que forman parte de estas, como política sectorial, política industrial, políticas económicas para el cambio del modelo productivo, entre las principales.

Para entender el contexto especifico de acción de la PEE, es pertinente reseñar el marco teórico relacionado a las CV y CL y dentro de estos, el análisis de la competitividad de las empresas y sectores industriales.

Porter (1985; 1990) desarrolla formalmente el concepto tradicional de CV y CL haciendo referencia en el primer caso de CV un modelo teórico, que detalla las actividades empresariales que una organización debe desarrollar, dentro de su proceso productivo, las mismas que permiten generar una propuesta de valor distintiva al cliente o grupo de clientes finales. Para el segundo, expresa que los CL son concentraciones geográficas de empresas e instituciones interconectadas en un campo en particular, las que abarcan una variedad de industrias vinculadas y otras entidades importantes para la competencia.

La PEE al tener como objetivo central el desarrollo y fomento productivo, debe priorizar los sectores de la economía, entendiendo la particularidad en las actividades económicas que estos tienen, en lo relacionado a su nivel de desarrollo real y potencial, de tal manera de priorizar políticas sectoriales (PS) que permitan el crecimiento de los ingresos y, a su vez, del nivel de gasto de los agentes económicos participantes de los procesos productivos. (Ver Figura 1).

Sánchez et al (2018) manifiesta que las políticas sectoriales se concentran en el aumento de la productividad, cuyo objetivo se relaciona al grado de desarrollo del país, la importancia del sector público, la política respecto al exterior, la estrategia de desarrollo, el grado de libertad de las inversiones extranjeras y la definición de las prioridades sectoriales.

De acuerdo con estos autores la transformación productiva depende entonces directamente de las prioridades de gobierno sobre las actividades y dinámicas propias que tienen los sectores de la economía. En este contexto específico, las políticas sectoriales (PS) toman relevancia ya que el fomento productivo se vincula directamente al desarrollo de CL y CV.

$\mathrm{Al}$ respecto de las políticas económicas para el cambio del modelo productivo, estas se orientan principalmente a las estrategias vinculadas al fomento de la investigación, desarrollo e innovación ( $\mathrm{I}+\mathrm{D}+\mathrm{I})$ cuya acción principal se orienta al potenciamiento de los factores productivos, mejora de la productividad y a través de esta, generar un impacto positivo en el crecimiento económico sectorial. Autores como Metcalfe (1995), Malerba (2002) y Andreoni (2018) enfatizan la necesidad de poder contar con una política I+D+i, bien definida y dentro de un sistema nacional de innovación y sentar las institucionales para el desarrollo de productivo. Las principales contribuciones que se reseñan en la literatura científica, coinciden en la concepción holística al respecto del proceso de I+D+I, esquema en el cual están integrados una red de actores claves vinculados al desarrollo productivo de los sectores económicos. 


\section{Competitividad y su relación con las políticas estructurales}

La idea central de la revisión del marco teórico es poder vincular los cambios institucionales y su relación con su incidencia en los niveles de competitividad y en ese contexto al desarrollo de los CL y las CVL.

En lo relacionado a la competitividad, el marco teórico ha evolucionado con enfoques que conectan la competitividad con nuevos trabajos sobre transformación estructural. En concordancia Lin (2011) ha realizado aportes concretos relacionando la importancia de las políticas orientadas a la transformación productiva y en igual sentido Rodrik (2004), Warwick, (2013), Stiglitz y Lin (2013) han enfatizado el enfoque de nueva política industrial y su relación positiva con la competitividad.

La literatura científica ha desarrollado enfoques complementarios cuyos alcances abordan investigaciones sobre complejidad económica (Hausmann y Klinger, 2007; Hausmann et al., 2013), geografía económica evolutiva (Boschma et al., 2017; Neffke et al., 2011) y sistemas de innovación (Nelson, 1993; Asheim y Gertler, 2005) coinciden en todos los casos, en lo relevante del enfoque y su incidencia en la evolución de la competitividad de un país.

Para fines del presente trabajo se tomará como base los indicadores analizados por el foro económico mundial (world economic fórum, por sus siglas en inglés), institución encargada de medir la competitividad de los países, a través del informe denominado reporte global de competitividad, reporte que se basa, esencialmente, en la medición de los llamados pilares de competitividad de una economía.

El índice de competitividad mide la capacidad de un país, para desarrollar altos niveles de bienestar económico, prosperidad y calidad de vida a sus ciudadanos. En consecuencia, el índice mide un conjunto de instituciones, políticas y factores que definen los niveles de bienestar y prosperidad económica que los países son capaces de desarrollar, sostener, e incrementar para aumentar el nivel de vida de sus habitantes y el desarrollo de sus economías. Las teorías sobre la competitividad nacional, es una de las áreas de estudio económico que ha tenido un gran crecimiento en su producción científica en los últimos 20 años.

\section{Principales teorías económicas aplicadas al fomento productivo.}

En este subapartado vamos a revisar los enfoques principales de la teoría macroeconómica aplicadas a las políticas de desarrollo productivo, políticas económicas estructurales, fomento de CL y CVL, las cuales incluyen importantes aportes que generan debates permanentes al respecto de las causas que explican el crecimiento económico.

Desde tiempos de Adam Smith (1776) el denominado padre de la economía, se indicaba que la riqueza de toda sociedad, el conjunto de bienes o valores de uso que satisfacen las necesidades y deseos de los individuos que la componen, tiene su origen en la producción, de tal manera que la riqueza y el bienestar social, se genera y se amplía a través de la actividad productiva.

Estos criterios siempre se orientaron a determinar la importancia de la producción, como la ventaja absoluta de Adam Smith (1776) ventaja comparativa de David Ricardo 
(1817) la teoría de la integración económica de Balassa (1965) el modelo Heckscher-Ohlin (1977) entre otros aportes importantes que tienen como denominador común, que todos estos estudios siempre se orientan a lo que un país es capaz de producir primero.

Esta idea se expone a cuestionamientos en épocas de crisis económicas, puesto que la gran depresión económica de 1929 originada en Estados Unidos, implicó una caída de la producción total de la economía norteamericana, trayendo consigo el efecto lógico reflejado en la caída de los ingresos y posteriormente del gasto en la economía.

En este contexto John Maynard Keynes (1936), desarrolla una serie de planteamientos cuya idea central se orienta a que las economías capitalistas no están limitadas por el lado de la oferta, sino, por el lado de la demanda, por esta razón, los niveles de desempleo, es consecuencia del gasto insuficiente en bienes y servicios. Keynes enfatiza en el papel de la demanda agregada para explicar el nivel y las fluctuaciones del producto generado en una economía.

Pero lo que Keynes denomina insuficiencia de la demanda agregada (gasto total o agregado de la economía) es el efecto visible de la caída previa de los niveles de ingreso de los agentes económicos y, esto a su vez, es la consecuencia lógica de la caída del producto total. Por lo tanto, el gasto insuficiente que la economía experimenta en un determinado momento del tiempo, no es más que la contraparte o espejo del decrecimiento de la producción en ese determinado momento del tiempo.

Para entender la lógica del argumento económico keynesiano (análisis de corto plazo) este se fundamenta en que se puede arreglar el problema central del crecimiento económico, atacando el efecto (demanda agregada,) y no la causa (oferta agregada), criterios que son contradictorios, puesto que un mayor consumo va en detrimento del ahorro y sin un nivel de ahorro no puede existir, a través de la función empresarial, un proceso económico de inversión que permita el mayor empleo de todos los factores productivos, para así incrementar la producción.

De acuerdo con Alonso-Neira, M (2004) existe un contraste entre la macroeconomía keynesiana (o del corto plazo) con el enfoque neoclásico basado en la economía del crecimiento de Robert Solow (o del largo plazo), ya que puede comprobarse que en el primer enfoque (corto plazo), las magnitudes de inversión y consumo se mueven en la misma dirección, siendo variables fuertemente procíclicas. Por el contrario, en el segundo enfoque (largo plazo), sólo es posible incrementar la inversión y, por tanto, el consumo futuro, si se renuncia a parte del consumo presente.

En el contexto de largo plazo, Solow (1956), plantea que para que un país pueda cambiar su estado estacionario a niveles de rentas per cápita más altos se deben realizar progresos técnicos, enfatizando la innovación tecnológica como factor clave para contrarrestar los rendimientos decrecientes, alcanzando así mayores niveles de producción, aún con la misma cantidad de capital y trabajo.

En base a esta contradicción entre el corto y largo plazo, Alonso-Neira, M (2004) plantea que: "ni la macroeconomía keynesiana, orientada hacia el presente, ni la macroeconomía neoclásica, con una clara orientación hacia el largo plazo, ofrecen una explicación satisfactoria de esta aparente contradicción”. 
Para complementar estos dos enfoques de corto y largo plazo, la teoría macroeconomía del capital, permite explicar el acoplamiento entre el corto y largo plazo. El origen de este enfoque tiene su origen en la escuela austriaca de economía, la cual destaca la naturaleza temporal del proceso de producción. Importantes economistas de esta escuela, como Ludwig Von Mises $(1912,1953)$ y F. Von Hayek (1929) realizarían importantes aportes a este esquema, como, por ejemplo, la idea de que el capital físico no es homogéneo, sino que está compuesto por un conjunto de factores de producción altamente desagregado y especializado.

Hayek (1996) argumenta como determinantes de la producción, los cambios en la estructura productiva, lo cual indica puede extenderse y volverse más compleja, cuanto más diversa sea la oferta de bienes y cuanto mayor sea la cantidad de bienes de órdenes superiores comprometidos en la generación de la oferta total. La lógica de estos argumentos radica en que las decisiones de inversión productiva tienen como contrapartida lógica, una estructura temporal del consumo, la cual determina las posibilidades de satisfacer las necesidades presentes y futuras.

Para complementar lo indicado por Hayek, Garrison (2001) en su obra "Tiempo y Dinero: La Macroeconomía de la Estructura del Capital" plantea tres instrumentos gráficos que actúan como bloques constitutivos de este enfoque, 1) el mercado de fondos prestables, 2) la frontera de posibilidades de producción (FPP), y 3) la estructura intertemporal de la producción o, lo que es igual, el triángulo de Hayek, (herramienta exclusiva de la macroeconomía del capital), esta obra enfatiza, la estructura intertemporal del capital (que al mismo tiempo refleja su heterogeneidad) y la subjetividad de las expectativas empresariales.

La revisión preliminar realizada sobre las teorías económicas principales aplicadas al fomento productivo, tienen como objetivo, una aproximación teórica que permita contribuir al entendimiento de la importancia de la producción como principal causa que explica el bienestar económico en una sociedad.

\section{Metodología}

Este trabajo tiene un enfoque cualitativo que se fundamenta en una investigación descriptiva cuyo principal objetivo es describir sistemáticamente los hechos y características del área de interés del presente trabajo. La investigación cualitativa a utilizar se fundamenta en una perspectiva interpretativa, que se centra en el entendimiento del fenómeno de estudio o análisis, evaluando principalmente el desarrollo de los sucesos que guardan relación a la evolución de la institucionalidad y los marcos regulatorios implementados en el Ecuador en el periodo de referencia entre los años 2007 - 2017.

El trabajo utiliza el método analítico cuyo fundamento está en la descomposición de un todo, estudiando sus partes o elementos para observar las causas, la naturaleza y los efectos que genera. De manera complementaria el método deductivo, el mismo que considera que la conclusión se halla implícita dentro las premisas, esto quiere decir, que las conclusiones son una consecuencia necesaria de las premisas.

La hipótesis de la que se parte en este estudio, se sustenta en que los indicadores de competitividad, crecimiento y desarrollo productivo, tienen una relación directa con los conceptos de institucionalidad y que existe una relación positiva entre cada uno de estos 
indicadores. Se considera al objeto de estudio, los cambios institucionales suscitados en el periodo de gobierno ecuatoriano entre los años 2007-2017 y su incidencia en la competitividad del Ecuador.

Este trabajo desarrolla un tipo de investigación descriptiva y aplicada, puesto que, parte desde la teoría para llegar a causalidades y conclusiones para explicar un problema práctico. El trabajo utiliza una metodología de investigación bibliográfica y exploratoria, cuyo eje central se fundamenta en el análisis de la información teórica existente y que se ha explicado en el marco teórico, complementándola con publicaciones periódicas y teorías relacionadas con el tema de estudio.

La organización del estudio tiene sus cimientos en dos etapas:

i. En primer lugar, la fase exploratoria, puesto que se buscó el descubrimiento de factores, indicadores y variables económicas importantes, como por ejemplo la incidencia de la institucionalidad en las actividades económicas, el desarrollo productivo y la competitividad de la economía ecuatoriana.

ii. En segundo lugar, se utilizó el nivel descriptivo para estudiar las relaciones y, correlaciones entre dichas variables, como por ejemplo el describir la relación entre los cambios institucionales suscitados en el periodo de referencia de estudio y su incidencia en la evolución de la competitividad del Ecuador en ese mismo periodo.

Para la recolección de los datos se tuvo como fuente primaria de información los informes, estudios y análisis de diferentes instituciones oficiales del país, como la secretaría nacional de planificación y desarrollo, banco central del Ecuador y asamblea nacional del Ecuador, la que se complementó con la información obtenida de los reportes globales de competitividad emitidos por el foro económico mundial.

\section{Modelo de política económica estructural}

En este trabajo se presenta un modelo teórico de análisis económico denominado "modelo o esquema de política económica estructural" (Ver Figura 1) el mismo que presenta los criterios sobre el potenciamiento del desarrollo productivo como base que permitiría el aumento de los ingresos de los agentes económicos participantes de dichas actividades y en base a estos ingresos se podrán tomar las decisiones de consumo y gasto (inducido) en la economía.

El esquema de política económica estructural plantea lo siguiente:

$$
P=Y=G(1)
$$

El nivel de producción (P) que una economía es capaz de desarrollar, generar, sostener o incrementar en el tiempo, será igual al nivel de ingreso y renta disponible (Y) que dicha economía será capaz de obtener y esta a su vez determinará la capacidad de gasto (inducido) presente y futuro $(\mathrm{G})$ que podrá realizar en el tiempo y es lo que permitirá a esta economía, financiar el consumo que necesitan sus familias, el financiamiento de la inversión que requieren sus empresas y de igual manera el nivel de gasto público que su estado requiere realizar para financiar su nivel de gasto corriente e inversión pública.

Esta obra se comparte bajo la licencia Creative Common Atribución-No Comercial 4.0 International (CC BY-NC 4.0)

Revista de la Universidad Internacional del Ecuador. URL: https://www.uide.edu.ec/ 


\section{Nivel de producción (P)}

El nivel de producción de una economía (P) (se relaciona directamente con el incremento o decremento de los niveles de producción) se vinculan directamente con la productividad generada en los sectores de la economía (Sector Primario, Sector Industrial y Sector Servicios), este nivel de producción estará en función de las decisiones intertemporales de consumo y ahorro disponible de la economía, que afectan positiva o negativamente a la inversión productiva, la oferta agregada, la producción real y potencial de la economía.

La productividad que una economía es capaz de desarrollar, se relaciona directamente con la condición de los factores productivos (FP) que posee, estos factores productivos son:

$$
F P=T \cdot(R N+t+K+O)(2)
$$

i. $\quad \mathrm{T}=$ Condición del nivel de tecnología existente disponible o restricciones tecnológicas para alcanzar un mayor aprovechamiento de la productividad que afecta al crecimiento. La tecnología se relaciona a las actividades de innovación, desarrollo e innovación (I+D+i) que afectan positivamente a los procesos productivos.

ii. $\quad \mathrm{RN}=$ Condición sobre la amplia dotación o escasez de recursos naturales, aspectos vinculados a la geografía económica, ubicación geográfica, clima y aspectos afines a las condiciones naturales que posee una economía.

iii. $\quad \mathrm{t}=$ Condición del trabajo, disponibilidad o restricción de trabajo muy calificado, semi calificado o poco calificado.

iv. $\quad \mathrm{K}=$ Condición del capital: disponibilidad, acceso o restricción a bienes de capital para la generación de producción, acceso a inversión directa extranjera.

v. $\mathrm{O}=$ Condición del nivel de organización empresarial, sofisticación de los procesos empresariales, redes empresariales de trabajo entre empresas, gobierno y universidades para potenciar el proceso productivo. innovación en procesos y modelos de negocios. 


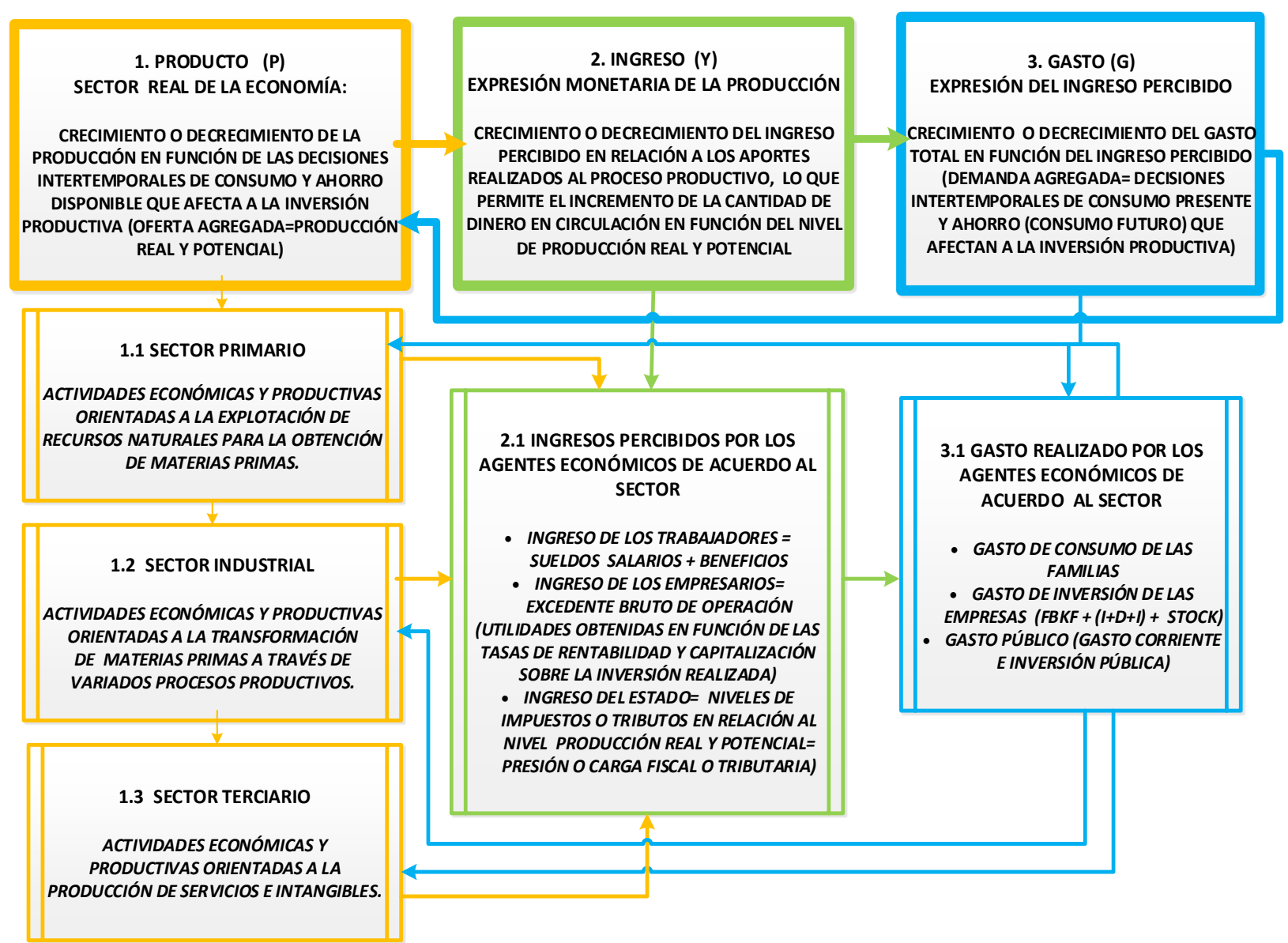

Figura 1 Modelo o esquema de política económica estructural, la misma que se presenta como un diagrama de causa y efecto, en el cual el fomento del desarrollo productivo como objetivo fundamental de las políticas económicas estructurales, es la base (causa) que genera los incrementos del ingreso y del gasto (efectos) en el sistema económico.

\section{Nivel de ingreso y renta disponible (Y)}

El nivel de renta e ingresos obtenidos en una economía (Y), se relacionan directamente con los aportes que los agentes económicos (familias, empresas y estado) realizan el proceso productivo y se vinculan de igual manera al incremento o decremento de los niveles de producción y a la productividad generada en los sectores de la economía.

El nivel de ingreso que los agentes económicos obtienen es la expresión monetaria de la producción, por lo tanto, hay que darle énfasis al entendimiento de que el crecimiento o decrecimiento del ingreso percibido, se relaciona directamente a dichos aportes al proceso productivo, lo que, a su vez, permite el incremento o decremento de la cantidad de dinero en circulación, en función directa del nivel de producción.

Los ingresos obtenidos se expresan de la siguiente forma:

$$
Y=S y S+B+E \cdot B \cdot O+U+T(3)
$$

Esta expresión hace referencia a los ingresos obtenidos por los trabajadores (YT) y los empresarios (YEM) a través de sus aportes al proceso productivo y los ingresos del estado 
obtenidos a través de los impuestos a las actividades económicas y la recaudación tributaria (T).

$$
Y T=S y S+B(4)
$$

Ingresos de los trabajadores (YT) es igual a los sueldos y salarios (S y S) más beneficios de los trabajadores que aportan en los sectores de la economía (B).

$$
Y E M=E . B . O(U)(5)
$$

Ingreso de los empresarios (YEM) es igual al excedente bruto de operación (E.B.O) que son los ingresos generados por el proceso de producción (principalmente utilidades (U) generadas por los empresarios obtenidas en función de tasas de rentabilidad y capitalización sobre la inversión realizada en los sectores de la economía).

$$
Y E S=T(6)
$$

Ingreso del Estado (YES) son los niveles de impuestos o tributos (T) en relación directa del nivel de producción real y potencial, lo que origina la presión o carga fiscal o tributaria para los sectores de la economía. Para efectos de simplificar el análisis dentro del modelo planteado, se asume que el único ingreso del estado, es en este caso, los impuestos que recauda de la actividad económica privada.

\section{Nivel de Gasto Agregado o Demanda Agregada (G)}

El nivel de gasto total o demanda agregada de una economía, es una expresión del ingreso percibido que se relaciona directamente a los aportes que los agentes económicos realizan al proceso productivo y se vinculan de igual manera al incremento o decremento de los niveles de producción y a la productividad generada en los sectores de la economía. Las decisiones de gasto se relacionan con las decisiones intertemporales de consumo y ahorro que afectan positiva o negativamente a la inversión productiva.

La demanda agregada de la economía se expresa de la siguiente forma:

$$
D A=C F+I+G P+(X-M)(7)
$$

Esta expresión hace referencia al gasto de las familias (CF) al gasto de inversión de las empresas (I) al gasto público (GP) del estado y a la interacción de la oferta y demanda de los mercados internacionales (X-M).

La composición de esta demanda agregada tiene la siguiente estructura:

$$
C F=C b y s N+C b y s E(8)
$$

Esta expresión se relaciona con la demanda y el consumo de bienes y servicios nacionales ( $\mathrm{C}$ b y s $\mathrm{N}$ ) realizados en los sectores de la economía nacional y/o la demanda y el consumo de bienes y servicios extranjeros $(\mathrm{C} \mathrm{b}$ y s $\mathrm{E})$ realizados en los sectores de la economía internacional. 


$$
I=F B K F+V E+(I+D+i)(9)
$$

Esta expresión se refiere al gasto de inversión (o reinversión) de las empresas realizados en los sectores de la economía, se relaciona a la formación bruta de capital fijo (FBKF) adquisición de maquinarias, activos fijos y variación de existencias (VE), se han incluido las actividades de investigación, desarrollo e innovación (I+D+i) para la generación de mayor producción.

$$
G=G C+I P(10)
$$

Esta expresión se refiere al gasto público (G) que es igual al gasto corriente (GC) e inversión pública (IP) realizado por el estado en los sectores de la economía, gasto que se relaciona directamente con el nivel de producción (real y potencial) e ingresos generados en los sectores de la economía, lo cual permite al estado realizar las asignaciones de gasto en sueldos, salarios del sector público para sustentar la provisión de bienes y servicios públicos, otorgamiento de subsidios y el fomento de planes de inversión pública orientados al fortalecimiento de la estructura productiva nacional, para así, cumplir con los objetivos de política económica.

$$
X-M=(O E N+O E E)-(D E+D I)(11)
$$

Esta expresión hace referencia a la oferta externa nacional (OEN) la cual satisface una demanda externa (DE) de bienes y servicios nacionales y a la oferta externa extranjera (OEE), la cual satisface una demanda interna (DI) de bienes y servicios internacionales.

El saldo (X-M) hace referencia a la interacción de oferta y demanda de los mercados internacionales, medida a través de:

i. La oferta externa de bienes y servicios nacionales (producción nacional orientada a mercados externos que se transforman en exportaciones nacionales) que satisfacen una demanda externa de dichos bienes y servicios nacionales (demanda externa de bienes y servicios que se transforman en importaciones extranjeras de la producción nacional).

ii. La oferta externa de bienes y servicios internacionales (producción internacional orientada a mercados externos que se transforman en exportaciones extranjeras) que satisface una demanda interna de dichos bienes y servicios internacionales (demanda interna de bienes y servicios extranjeros que se transforman en importaciones nacionales de productos extranjeros).

El modelo enfatiza la importancia de los cambios en la estructura productiva como base del desarrollo y recalcar que el nivel de gasto (inducido), es una expresión del nivel de ingreso percibido, que se convertirá finalmente en la demanda agregada o global de la economía.

Es en este contexto de análisis, donde en Ecuador surgen políticas económicas estructurales orientadas a la transformación productiva y el desarrollo de los clústeres locales (CL) y las cadenas de valor local (CVL) como eje de los cambios lógicos que deberían incidir en las mejoras de la productividad y sus efectos en la dinámica económica de generación de ingresos e incremento del nivel de gasto total de la economía 


\section{Resultados y discusión}

En el periodo 2007-2017 en Ecuador el gobierno nacional implementó diversos cambios institucionales que abarcaron aspectos políticos, legales, económicos, financieros, los mismos que han contribuido a generar resultados que mejoraron el crecimiento de la economía. Estas reformas iniciaron con una serie de cambios institucionales en la creación de una nueva asamblea nacional constituyente que promulgó una nueva constitución en el año 2008 cuyo objetivo era encaminar al país hacia un nuevo orden económico-social.

Esta nueva constitución introdujo reformas hacia temas relacionados a la agenda nacional de desarrollo y cuestiones ligadas a la recuperación de la planificación pública, la construcción de un estado constitucional que garantice derechos a los ciudadanos. Las reformas institucionales en este campo implicaron aclarar las competencias, facultades y atribuciones en la administración pública central y en los distintos niveles de gobierno para el logro de los objetivos relacionados al desarrollo productivo. (Ver en Anexos Tabla 1).

\section{Resultados económicos en materia de competitividad e institucionalidad derivados de los cambios institucionales implementados}

El presente trabajo sostiene que las bases institucionales que un país es capaz de generar y desarrollar, son factores que condicionan de manera positiva el poder alcanzar resultados económicos que permitan el crecimiento y desarrollo económico.

En función de este concepto, se va a desarrollar una revisión de los principales resultados económicos obtenidos en el Ecuador en materia de competitividad y que guardan estrecha relación con la implementación de los cambios institucionales y marcos jurídicosadministrativos creados para incidir positivamente en el fomento y desarrollo productivo de la economía ecuatoriana.

Tabla. 2

Ecuador: Evolución de la Competitividad Global

\begin{tabular}{ccc}
\hline RGC & $\begin{array}{c}\text { POSICIÓN COMPETITIVA } \\
\text { DEL ECUADOR }\end{array}$ & $\begin{array}{c}\text { TOTAL DE PAISES } \\
\text { ESTUDIADOS }\end{array}$ \\
\hline INDICE 2007-2008 & 103 & 134 \\
INDICE 2008-2009 & 104 & 134 \\
INDICE 2009-2010 & 105 & 133 \\
INDICE 2010-2011 & 105 & 139 \\
INDICE 2011-2012 & 101 & 142 \\
INDICE 2012-2013 & 86 & 144 \\
INDICE 2013-2014 & 71 & 148 \\
INDICE 2014-2015 & AUSENTE & AUSENTE \\
INDICE 2015-2016 & 76 & 140 \\
INDICE 2016-2017 & 91 & 138 \\
INDICE 2017-2018 & 97 & 137 \\
\hline \multicolumn{2}{c}{ Fuente: Reporte Global de Competitividad, Foro Económico Mundial } \\
& Elaboración Propia.
\end{tabular}

Es importante anotar que la evolución competitiva del Ecuador es favorable hasta el reporte del año 2013-2014, cuando se ubicó en el puesto 71 entre 140 economías a nivel mundial un mejoramiento competitivo de 31 puestos con respecto al periodo 2007-2008, es a Esta obra se comparte bajo la licencia Creative Common Atribución-No Comercial 4.0 International (CC BY-NC 4.0) Revista de la Universidad Internacional del Ecuador. URL: https://www.uide.edu.ec/ 
partir del siguiente periodo que inicia un retroceso en el índice de competitividad global, iniciando con un descenso de 5 puestos para el periodo 2015-2016 y acentuándose su caída para los siguientes periodos 2016-2017 y 2017-2018, con un retroceso de 21 puestos en su posición competitiva, ya que para el último reporte 2017-2018, el país estuvo en el puesto 97 entre 137 economías analizadas.

Estos resultados muestran dos etapas, una primera etapa con grandes mejorías en la posición competitiva del Ecuador, lo cual se ve reflejado hasta el periodo 2013-2014 y luego de una etapa de reformas legales e institucionales que fueron generando condiciones favorables para la competitividad de la economía ecuatoriana. Este mejoramiento nos lleva a determinar la importancia de las reformas legales e institucionales que el Ecuador fue implementado en los primeros años del periodo analizado.

Hay que recalcar que estos cambios institucionales y la priorización de estas políticas de reforma, se realizan en un contexto económico favorable para la economía de este país, sustentado principalmente en altos precios internacionales del petróleo, que por una parte favorecen el ingreso de divisas por concepto de exportaciones petroleras, cuyo ingreso considerado como ingreso no permanente, permite financiar el gasto público y las inversiones del estado como agente clave que asume el rol dinamizador de la economía, pero por otra parte, ponen en evidencia, un alto nivel de dependencia y de vulnerabilidad de la economía, ante la volatilidad de los precios del hidrocarburo, situación que se pone de manifiesto, para los periodos siguientes de análisis.

Realizando una breve síntesis de la evolución, las 31 posiciones que el país logró alcanzar entre los años 2011 y 2013 no se pudieron sostener, cuando la economía ecuatoriana se vio expuesta a un shock externo negativo, que fue la caída internacional de los precios del petróleo que generó una desaceleración y recesión de la economía, teniendo un retroceso y una pérdida de 26 posiciones.

Tabla. 3

Ecuador: Pilar de Competitividad Institucional

\begin{tabular}{ccc}
\hline RGC & $\begin{array}{c}\text { POSICIÓN COMPETITIVA DEL } \\
\text { ECUADOR }\end{array}$ & $\begin{array}{c}\text { TOTAL DE PAISES } \\
\text { ESTUDIADOS }\end{array}$ \\
\hline INDICE 2007-2008 & 125 & 134 \\
INDICE 2008-2009 & 129 & 134 \\
INDICE 2009-2010 & 125 & 133 \\
INDICE 2010-2011 & 128 & 139 \\
INDICE 2011-2012 & 125 & 142 \\
INDICE 2012-2013 & 131 & 144 \\
INDICE 2013-2014 & 92 & 148 \\
INDICE 2014-2015 & AUSENTE & AUSENTE \\
INDICE 2015-2016 & 105 & 140 \\
INDICE 2016-2017 & 113 & 138 \\
INDICE 2017-2018 & 128 & 137 \\
\hline
\end{tabular}

Fuente: Reporte Global de Competitividad, Foro Económico Mundial Elaboración Propia.

Entre los principales factores que evidencian las mejoras en la posición competitiva del Ecuador que se vio hasta el periodo 2011-2013, se encuentran el rediseño de la estructura institucional del estado, la infraestructura nacional, el sistema educativo, la innovación y la Esta obra se comparte bajo la licencia Creative Common Atribución-No Comercial 4.0 International (CC BY-NC 4.0) Revista de la Universidad Internacional del Ecuador. URL: https://www.uide.edu.ec/ 
capacidad tecnológica del país. Los cambios institucionales nacieron de la promulgación de la nueva constitución promulgada en el año 2008, cuerpo legal que otorga a la planificación económica central, realizada por el estado, el rol clave en la agenda de cambio institucional e introduce el marco de acción para el desarrollo de un nuevo marco legal e institucional para el sistema económico ecuatoriano.

Debido a las profundas transformaciones políticas llevadas a cabo desde el año 2007, se evidencian los efectos positivos en la posición competitiva del Ecuador en materia de competitividad institucional, indicador que reflejó un notorio avance entre los años 20122013 y 2014, periodo en el cual, tuvo un avance de 39 puestos en su posición competitiva en el denominado pilar institucional. Para los siguientes periodos del 2015-2016 y 2017-2018 hay nuevamente un retroceso significativo de 36 puestos en el referido índice, justamente en el periodo donde la economía se ve expuesta a shocks económicos y queda en evidencia su vulnerabilidad.

\section{Aspectos generales sobre la competitividad global del ecuador}

La competitividad global del Ecuador en estos 10 años ha tenido en promedio una evolución favorable, tomando como base las posiciones competitivas en los diferentes reportes anuales y comparándolos con el periodo 2007-2008, donde se inicia este estudio. De acuerdo con esto se puede ver la evolución positiva sobre el desempeño de la economía del Ecuador, teniendo un evidente avance del país en materia de competitividad global, ya que se muestran notables progresos en las mejoras de la competitividad del país principalmente para el periodo 2013-2014 y 2015-2016, donde el Ecuador se ubicó en los puestos 71 y 76 entre 148 y 140 países respectivamente en comparación con el puesto 103 cuando inicia el índice en el periodo 2007-2008.

Tabla. 4

Ecuador: Pilar Estabilidad Macroeconómica

\begin{tabular}{ccc}
\hline \multicolumn{1}{c}{ REPORTE } & $\begin{array}{c}\text { POSICIÓN COMPETITIVA DEL } \\
\text { ECUADOR }\end{array}$ & $\begin{array}{c}\text { TOTAL DE PAISES } \\
\text { ESTUDIADOS }\end{array}$ \\
\hline INDICE 2007-2008 & 27 & 134 \\
INDICE 2008-2009 & 16 & 134 \\
INDICE 2009-2010 & 44 & 133 \\
INDICE 2010-2011 & 55 & 139 \\
INDICE 2011-2012 & 40 & 142 \\
INDICE 2012-2013 & 37 & 144 \\
INDICE 2013-2014 & 44 & 148 \\
INDICE 2014-2015 & AUSENTE & AUSENTE \\
INDICE 2015-2016 & 75 & 140 \\
INDICE 2016-2017 & 83 & 138 \\
INDICE 2017-2018 & 91 & 137 \\
\hline \multicolumn{2}{c}{ Fuente: Reporte Global de Competitividad, Foro Económico Mundial }
\end{tabular}

Los factores que evidencian las mejoras, están determinados y explicados principalmente por la incidencia de la política pública del gobierno nacional en materia de inversiones públicas enfocadas al desarrollo de carreteras, puertos, aeropuertos, políticas 
educativas a nivel de salud y educación primaria; educación superior y el fomento a las actividades relacionadas a la investigación y el desarrollo de la capacidad tecnología del país.

Es importante recalcar que para poder analizar integralmente la pertinencia financiera de las inversiones públicas realizadas se debería de analizar técnicamente la estructura óptima de financiamiento de las referidas inversiones públicas (en función de criterios claves como la vida útil del activo a ser financiado y su relación con el plazo de la deuda contratada y la tasa de interés a pagar por la misma). El objetivo de este análisis es determinar la capacidad de pago del estado sobre las inversiones realizadas, que están sosteniendo las mejoras en el índice de competitividad, para poder inferir si es sostenible la política de fomento productivo.

\section{Estabilidad macroeconómica}

Para mediados del año 2015 y hasta mediados del año 2016, la caída internacional de los precios del petróleo, la apreciación del dólar norteamericano 1 ha generado graves efectos para la economía ecuatoriana, con un efecto visible en el sector fiscal (ingresos fiscales en caída) en el sector externo (efectos negativos en la balanza de pagos) y el sector real (sectores productivos deprimidos). Todo esto ha incidido negativamente en el indicador de competitividad en lo relacionado a la estabilidad macroeconómica, donde el país retrocede al puesto 75 entre 150 países, retrocediendo 31 puestos con respecto al último reporte de competitividad global 2013-2014.

La dependencia de la economía ecuatoriana a los ingresos por exportaciones de petróleo, es el factor que afectó negativamente a la economía en su conjunto, puesto que la caída internacional de los precios del petróleo, se combinó entre los años 2015 y 2016, con la apreciación del dólar estadounidense en los mercados internacionales y a la devaluación de las monedas de los países vecinos, países exportadores de productos similares a los del Ecuador, lo que afecto a las exportaciones no petroleras y agravó la situación económica, generando decrecimiento económico, caída de los ingresos de todos los agentes de la economía, incrementos de los indicadores de desempleo y subempleo, sumados a una contracción de la actividad económica en general, lo que ha contribuido al retroceso en varios indicadores de competitividad.

La relación causa-efecto que ha contribuido a este escenario actual se puede resumir en los siguientes puntos:

i. Caída de los precios internacionales del petróleo ecuatoriano importante ingreso de exportación que financia el presupuesto general del estado y las decisiones de gasto e inversión pública, el país deja de recibir ingresos importantes por divisas y por lo tanto esto afecta directamente las decisiones de política fiscal.

ii. Apreciación del dólar en los mercados financieros internacionales y depreciación de monedas de países latinoamericanos, exportadores de productos similares a los del ecuador, debido a esto, pérdida de competitividad en el sector exportador de varios productos (atún, flores, café, cacao, entre otros etc.), reducción de exportaciones, pérdida de ingresos por divisas.

\footnotetext{
${ }^{1}$ Ecuador adoptó el dólar estadounidense como moneda de curso legal a partir del año 2000, constituyéndose en el sistema monetario vigente, el mismo que no le permite contar con instrumentos de política monetaria y cambiaria para ajustar la economía ante un desajuste del sector externo o la presencia de un shock económico interno, tal como sucedió entre los años 2015 y 2016.
} 
iii. Se pierden ingresos de divisas estimados en más de 12.000 millones de dólares un 12\% del Producto Interno Bruto PIB del año 2015 en la economía en su conjunto.

iv. Reducción de los depósitos en el sector financiero-bancario.

v. Medidas de ajuste del gasto e inversión pública, (reducción de personal en instituciones públicas, recortes de planes de inversión y contratación de servicios al sector privado)

vi. Medidas de ajustes del gasto e inversión privada reducción de personal en empresas privadas, recortes de planes de inversión y contratación de servicios)

vii. Reducción de las recaudaciones fiscales por parte del gobierno.

viii. Incremento del desempleo, reducción del consumo privado y afectación a la producción de bienes y servicios.

ix. Terremoto que afectó al país y género pérdidas económicas cuantiosas.

x. Pérdida de competitividad en varios sectores de la economía.

xi. Desaceleramiento y recesión económica.

De igual manera, es importante resaltar que el país retrocede en muchos de los pilares de competitividad, como el de sofisticación de los negocios pasando del puesto 69 en el periodo 2013-2014 al puesto 87 para el periodo 2015-2016, con un retroceso de 18 puestos, retroceso que se ve acentuado para el último reporte 2017-2018, donde el Ecuador cae a la posición 105 , lo que refleja una caída de similar de 18 puestos, acumulando un retroceso de 36 puestos en los últimos dos años.

Así mismo, el pilar de innovación tiene un retroceso de 28 puestos, pasando del puesto 58 en el periodo 2013-2014 al puesto 86 para el periodo 2015-2016. De igual manera para los siguientes dos reportes, esta caída se sostiene y pasa del puesto 86 hasta el puesto 117 para el periodo 2017-2018.

\section{Conclusiones}

Los resultados nos llevan a concluir que, de acuerdo índice de competitividad global del foro económico mundial, los cambios institucionales incidieron positivamente, en una primera etapa, en el mejoramiento competitivo de 31 puestos en la posición del Ecuador en el periodo 2013-2014 con respecto al periodo 2007-2008.

Posterior al reporte de 2013-2014 y hasta el periodo 2017-2018 se puede evidenciar un retroceso significativo de 26 posiciones en el referido reporte, periodo en el cual se evidencia la vulnerabilidad y dependencia en su estructura productiva cuando se ve expuesta a dos shocks económicos (Caída en el segundo semestre del año 2015 de los precios internacionales del petróleo y en Abril del 2016 la presencia de un terremoto, desastre natural) los cuales desencadenaron y agudizaron la recesión económica al no contar el estado con políticas económicas de respuesta para actuar ante estos shocks económicos (Estabilizadores Fiscales automáticos: Fondos Soberanos de Ahorro, Ejemplo petroleros y/o Seguros contra desastres naturales).

Es importante señalar que el mejoramiento en los indicadores de competitividad de la economía ecuatoriana durante el periodo de referencia 2007-2017, se van dando de manera gradual y progresiva, empezando por el ordenamiento del marco legal, cambio de reformas institucionales que contribuyeron a generar las condiciones favorables para al fomento de una política de desarrollo productivo. 


\section{Bibliografía}

Alonso Neira, M (2004). La macroeconomía del capital en el siglo XXI. Análisis Económico Núm. 40, vol. XIX Primer cuatrimestre de 2004.

Acemoglu D \& Robinson J. (2012). Por qué fracasan los países - Los orígenes del poder, la prosperidad y la pobreza. Bogotá, Editorial Planeta Colombiana.

Asheim, B., \& Gertler, M. S. (2005). The Geography of Innovation: Regional Innovation Systems. In J. Fagerberg, D. C. Mowery, \& R. R. Nelson (Eds.), The Oxford Handbook of Innovation (pp. 291-317). Oxford University Press.

Andreoni, A., (2018). The architecture and dynamics of industrial ecosystems: diversification and innovative industrial renewal in Emilia Romagna. Cambridge J. Econ. 42, 6.

Boschma et al (2017), Towards a theory of regional diversification: combining insights from Evolutionary Economic Geography and Transition Studies, Regional Studies, Vol. 51, No. 1, 31-45.

Caballero, G. \& Garza, M. (2003). "Los Fundamentos de la Nueva Economía Institucional hacia la Economía de los Recursos naturales: Comunes, Instituciones, Gobernanza y Cambio Institucional".

Garrison, Roger W. (2005) [2001], Tiempo y Dinero. La Macroeconomía de la Estructura del Capital, Unión Editorial, Madrid.

Hausmann, R et al (2013), The Atlas of Economic Complexity: Mapping Paths to Prosperity, Harvard University/MIT: Cambridge, MA.

Hausmann, R, Klinger B, (2007), The Structure of the Product Space and the Evolution of Comparative Advantage, CID Working Paper No. 146, Harvard Kennedy School of Government: Cambridge, MA.

Hayek von F. (1996 [1931]). Prices and Production. Londres: Routledge y Sons; Precios y Producción, trad. de [1931], Madrid: Unión Editorial.

Keynes, J. M. (1936). The General Theory of Employment, Interest and Money. New York: Polygraphic Company of America.

Lin, J. Y. (2011). New Structural Economics: A Framework for Rethinking Development. The World Bank Research Observer, 193-221.

Malerba, F., 2002. Sectoral system of innovation and production. Res. Policy 31,247-264.

Metcalfe, S., 1995. Technology systems and technology policy in an evolutionary framework. Cambridge J. Econ. 19, 25-46.

Neffke et al (2011), How Do Regions Diversify over Time? Industry Relatedness and the Development of New Growth Paths in Regions, Economic Geography, Vol. 87, 237265.

Nelson, R. (1993), National Innovation Systems: A Comparative Analysis, Oxford University Press: Oxford.

North, Douglass C. (1990). Institutions, Institutional Change and Economic Performance, Cambridge University Press.

Porter, M. (1985). Competitive Advantage: Creating and Sustaining a superior performance. New York: Free Press.

Porter, M.E. (1990) The Competitive Advantage of Nations. New York: Free Press.

Ricardo, D. (1985). Principios de economía política y tributación. Barcelona, España: Ediciones ORBIS.

Rodrik, D, (2004), Industrial Policy for the 21st Century, KSG Faculty Research Working Paper Series, RWP04-047, Harvard Kennedy School of Government: Cambridge, MA.

Sánchez A, Ochando C, Torrejón M (2018) Política económica estructural. Editorial: Universitat Oberta de Catalunya. (UOC). 
Smith, A. (1983). Una investigación sobre la naturaleza y causa de la riqueza de las naciones. Barcelona, España: Ediciones ORBIS.

Stiglitz, J., Lin J, 2013 (eds.). The Industrial Policy Revolution I: The Role of Government Beyond Ideology, Palgrave Macmillan: New York, NY.

Solow, R. (1956). A Contribution to the Theory of Economic Growth. The Quarterly Journal of Economic, 65-94.

Warwick, K, (2013), Beyond Industrial Policy: Emerging Issues and Trends, OECD Science, Technology and Industry Policy Papers, No. 2, OECD: Paris.

World Economic Forum. (2007) The Global Competitiveness Report 2007-2008. Professor Klaus Schwab, World Economic Forum Editor.

World Economic Forum. (2008) The Global Competitiveness Report 2008-2009. Professor Klaus Schwab, World Economic Forum Editor.

World Economic Forum. (2009) The Global Competitiveness Report 2009-2010. Professor Klaus Schwab, World Economic Forum Editor.

World Economic Forum. (2010). The Global Competitiveness Report 2010-2011. Professor Klaus Schwab, World Economic Forum Editor.

World Economic Forum. (2011). The Global Competitiveness Report 2011-2012. Professor Klaus Schwab, World Economic Forum Editor.

World Economic Forum. (2012). The Global Competitiveness Report 2012-2013. Professor Klaus Schwab, World Economic Forum Editor.

World Economic Forum. (2013). The Global Competitiveness Report 2013-2014. Professor Klaus Schwab, World Economic Forum Editor.

World Economic Forum. (2014). The Global Competitiveness Report 2014-2015. Professor Klaus Schwab, World Economic Forum Editor.

World Economic Forum. (2015). The Global Competitiveness Report 2015-2016. Professor Klaus Schwab, World Economic Forum Editor.

World Economic Forum. (2016). The Global Competitiveness Report 2016-2017. Professor Klaus Schwab, World Economic Forum Editor.

World Economic Forum. (2017). The Global Competitiveness Report 2017-2018. Professor Klaus Schwab, World Economic Forum Editor.

\section{Anexos}

Tabla.1

Principales leyes aprobadas en Ecuador periodo 2007-2017 en materia económica, productividad, empleo y competitividad

\begin{tabular}{l}
\hline Área de Política Económica \\
\hline Código Orgánico Monetario y Financiero. \\
\hline Código Orgánico de Planificación y Finanzas Públicas. \\
\hline Ley de Creación de la Red de Justicia Financiera. \\
\hline Ley Reformatoria a la Ley de Régimen Tributario Interno y a la Ley Reformatoria para la Equidad \\
Tributaria del Ecuador. \\
\hline Ley Orgánica para el Fortalecimiento y Optimización del Sector Societario y Bursátil. \\
\hline Ley Orgánica para el Cierre de la Crisis Bancaria de 1999. \\
\hline Ley de Economía Popular y Solidaría. \\
\hline Área de Producción, Empleo y Competitividad \\
\hline Ley Orgánica de Regulación y Control del Poder de Mercado. \\
\hline Código Orgánico de la Producción Comercio e Inversiones. \\
\hline Ley de Incentivos a la Producción y Prevención del Fraude Fiscal. \\
\hline
\end{tabular}


Ley Orgánica de Incentivos para el Sector Productivo.

Ley Orgánica de Incentivos para Asociaciones Público-Privadas y la Inversión Extranjera.

Ley Orgánica de Empresas Públicas.

Ley Reformatoria a la Ley para Reprimir el Lavado de Activos.

Área Sectores Estratégicos

Ley Reformatoria a la Ley de Hidrocarburos y a la Ley Orgánica de Régimen Tributario Interno.

Ley de Minería.

Ley de Fomento Ambiental y Optimización de los Ingresos del Estado.

Área Educación-Conocimiento

Ley Orgánica de Educación Superior.

Ley Orgánica de Educación Intercultural.

Fuente: Asamblea Nacional del Ecuador

Elaboración Propia.

Tabla. 5

Ecuador: Pilar de Sofisticación Empresarial

\begin{tabular}{ccc}
\hline \multicolumn{1}{c}{ REPORTE } & $\begin{array}{c}\text { POSICIÓN COMPETITIVA DEL } \\
\text { ECUADOR }\end{array}$ & $\begin{array}{c}\text { TOTAL DE PAISES } \\
\text { ESTUDIADOS }\end{array}$ \\
\hline INDICE 2007-2008 & 93 & 134 \\
INDICE 2008-2009 & 99 & 134 \\
INDICE 2009-2010 & 105 & 133 \\
INDICE 2010-2011 & 107 & 139 \\
INDICE 2011-2012 & 93 & 142 \\
INDICE 2012-2013 & 94 & 144 \\
INDICE 2013- 2014 & 69 & 148 \\
INDICE 2014-2015 & AUSENTE & AUSENTE \\
INDICE 2015-2016 & 87 & 140 \\
INDICE 2016-2017 & 87 & 138 \\
INDICE 2017-2018 & 105 & 137 \\
\hline
\end{tabular}

Fuente: Reporte Global de Competitividad, Foro Económico Mundial Elaboración Propia.

Tabla. 6

Ecuador: Pilar de Innovación

\begin{tabular}{ccc}
\hline REPORTE & $\begin{array}{c}\text { POSICIÓN COMPETITIVA DEL } \\
\text { ECUADOR }\end{array}$ & TOTAL DE PAISES ESTUDIADOS \\
\hline INDICE 2007-2008 & 118 & 134 \\
INDICE 2008-2009 & 129 & 134 \\
INDICE 2009-2010 & 129 & 133 \\
INDICE 2010-2011 & 130 & 139 \\
INDICE 2011-2012 & 110 & 142 \\
INDICE 2012-2013 & 94 & 144 \\
INDICE 2013-2014 & 58 & 148 \\
INDICE 2014-2015 & AUSENTE & AUSENTE \\
INDICE 2015-2016 & 86 & 140 \\
INDICE 2016-2017 & 107 & 138 \\
INDICE 2017-2018 & 111 & 137 \\
\hline
\end{tabular}

Fuente: Reporte Global de Competitividad, Foro Económico Mundial

Elaboración Propia.

Esta obra se comparte bajo la licencia Creative Common Atribución-No Comercial 4.0 International (CC BY-NC 4.0)

Revista de la Universidad Internacional del Ecuador. URL: https://www.uide.edu.ec/ 